\title{
Padovan sequence generalization - a study of matrix and generating function
}

\section{Renata Passos Machado Vieira ${ }^{1}$, Francisco Regis Vieira Alves ${ }^{2}$ and Paula Maria Machado Cruz Catarino ${ }^{3}$}

${ }^{1}$ Department of Mathematics, Institute Federal of Tecnology of State of Ceara (IFCE) Fortaleza-CE, Brazil e-mail: re.passosmegmail.com

${ }^{2}$ Department of Mathematics, Institute Federal of Tecnology of State of Ceara (IFCE) Fortaleza-CE, Brazil e-mail: fregis@gmx.fr

${ }^{3}$ Department of Mathematics, University of Trás-os-Montes and Alto Douro Vila Real, Portugal e-mail: pcatarino23@gmail.com

Received: 27 December 2019 Revised: 4 November 2020 Accepted: 9 November 2020

\begin{abstract}
The Padovan sequence is a sequence similar to the Fibonacci sequence, the former being third order and the latter second. Having several applications in architecture, these numbers are directly related to plastic numbers. In this paper, the Padovan sequence is studied and investigated from the standpoint of linear algebra. With this, we will study the matrix and the generating function of the extensions of this sequence (Tridovan and Tetradovan), thus determining the generalization of this sequence.
\end{abstract}

Keywords: Generating function, Generalization, Generator matrix, Padovan sequence. 2010 Mathematics Subject Classification: 11B36, 11B39.

\section{Introduction}

The Padovan sequence, considered a Fibonacci Sequence cousin, named after the Italian architect Richard Padovan (born in 1935), is a third order linear and recurring sequence of integers. Richard Padovan was born in the city of Padua [15], a little distant village of Pisa [1]. 
Just as the Fibonacci sequence has many applications in science, economics, biology and architecture [12], this sequence also has several interesting properties and applications in the fields of architecture, as reported by Padovan [11] and Voet and Schoonjans [19]. The Padovan sequence is defined by the following recurrence relationship:

$$
P_{n}=P_{n-2}+P_{n-3},
$$

with $P_{0}=P_{1}=P_{2}=1, n \geqslant 3$ and $P_{n}$ being the $n$-th term of the sequence.

This work considers the shifted Padovan sequence, presenting as initial values $P_{0}=1, P_{1}=$ $0, P_{2}=1$.

It can be seen that every linear sequence has a characteristic equation obtained from its constant recurrence coefficients, as studied in the work of Gomes [7]. The same is true for Padovan sequence and can be obtained from Equation (1).

Based on the Cayley-Hamilton theorem, Padovan's characteristic polynomial is given by [6]:

$$
p(\lambda)=\operatorname{det}(\lambda I-Q),
$$

with $\lambda \in \mathbb{Z}$ or $\lambda \in \mathbb{C}, \lambda I=\left[\begin{array}{lll}\lambda & 0 & 0 \\ 0 & \lambda & 0 \\ 0 & 0 & \lambda\end{array}\right]$ and $Q=\left[\begin{array}{lll}0 & 1 & 0 \\ 1 & 0 & 1 \\ 1 & 0 & 0\end{array}\right]$. Likewise:

$$
p(\lambda)=\operatorname{det}\left(\left[\begin{array}{ccc}
\lambda & -1 & 0 \\
-1 & \lambda & -1 \\
-1 & 0 & \lambda
\end{array}\right]\right)=\lambda^{3}-\lambda-1 \text {. }
$$

So: $p(\lambda)=0$, we have: $\lambda^{3}-\lambda-1=0$. Validating and defining the characteristic equation as:

$$
x^{3}-x-1=0,
$$

its roots are given by one real and two complex conjugates, namely:

$$
\begin{gathered}
x_{1}=\sqrt[3]{\frac{1}{2}+\frac{1}{6} \sqrt{\frac{23}{3}}}+\sqrt[3]{\frac{1}{2}-\frac{1}{6} \sqrt{\frac{23}{3}}} \\
x_{2}, x_{3}=\left(-\frac{1}{2} \pm \frac{\sqrt{3}}{2} i\right) \sqrt[3]{\frac{1}{2}+\frac{1}{6} \sqrt{\frac{23}{3}}}+\left(-\frac{1}{2} \mp \frac{\sqrt{3}}{2} i\right) \sqrt[3]{\frac{1}{2}-\frac{1}{6} \sqrt{\frac{23}{3}}} \approx-0.662359 \pm 0.56228 i
\end{gathered}
$$

The only real root found from this equation is known as the plastic number $\psi$, which represents the convergence relationship between the neighboring numbers of this sequence [5]. The plastic constant was first studied in 1924 by the French Gérard Cordonnier [20], and being defined by:

$$
\lim _{n \rightarrow \infty} \frac{P_{n+1}}{P_{n}}=\psi \approx 1,32 .
$$

This paper aims to discuss and generalize the matrix and the generating function of the Padovan sequence. A new approach to these extensions is based on the work of Vieira and Alves [17], introducing the Tridovan and Tetradovan numbers, respectively being fourth and fifth 
order sequences. Thus, by investigating the construction of these matrices and their generating function, which are other ways of obtaining the numbers of a sequence without using their recursion, we will primarily in this work start this study in order to generalize the sequence.

Finally, based on the Fibonacci sequence $[8,14,16]$, the Padovan sequence characteristic equation of any order will be generalized, thus allowing to easily obtain the equation, matrix and generator function of that sequence according to its order.

\section{Padovan sequence extensions}

Following the Vieira and Alves' works [17, 18], where the Padovan sequence extension for fourth (Tridovan) and fifth (Tetradovan) order are defined, it is possible to define a generalized sequence of any order.

Now a new sequence called the $Z$-dovan Sequence will be introduced, which is the generalization of the Padovan sequence extension of order $z$ and $Z=z-1$.

Definition 2.1. Tridovan sequence has the following recurrence formula, for $n \in \mathbb{N}$ [17]:

$$
P_{3(n)}=P_{3(n-2)}+P_{3(n-3)}+P_{3(n-4)},
$$

$n \geqslant 4$, where $P_{3(n)}$ represents the $n$-th term of the sequence with initial terms $P_{3(0)}=1, P_{3(1)}=0$, $P_{3(2)}=P_{3(3)}=1$.

Definition 2.2. Tetradovan sequence has recurrence relationship, with $n \in \mathbb{N}$ [17]:

$$
P_{4(n)}=P_{4(n-2)}+P_{4(n-3)}+P_{4(n-4)}+P_{4(n-5)},
$$

$n \geqslant 5$, where $P_{4(n)}$ represents the $n$-th term of the sequence with the initial terms $P_{4(0)}=1$, $P_{4(1)}=0, P_{4(2)}=P_{4(3)}=1, P_{4(4)}=2$.

Following the same idea of Tridovan (Definition 2.1) and Tetradovan (Definition 2.2), one can achieve the $Z$-dovan sequence as follows.

Definition 2.3. The Z-dovan sequence recurrence formula is given by:

$$
P_{Z(n+z)}=\sum_{i=0}^{z-2} P_{Z(n+i)},
$$

$n \geqslant z, n \in \mathbb{N}$, where $P_{Z(n)}$ represents the $n$-th sequence term. The initial terms of this sequence are: $P_{Z(0)}=1, P_{Z(1)}=0, P_{Z(2)}=P_{Z(3)}=1, P_{Z(4)}=2, \ldots$, where the amount of initial terms is defined by the sequence order, so for an sequence order $z$, z initial terms will be required and $Z=z-1$.

Every sequence has a characteristic equation, we will explore the Padovan, Tridovan, and Tetradovan sequence equations to define the $Z$-dovan polynomial.

For the Padovan sequence, we have the $x^{3}-x-1=0$ polynomial, shown at the beginning of this paper. For Tridovan we have the $x^{4}-x^{2}-x-1=0$ polynomial and for Tetradovan we have $x^{5}-x^{3}-x^{2}-x-1=0$ [17], where these demonstrations can be performed analogously to the validation developed for Padovan's characteristic equation (see Equation 2). 
Thus, the $(Z-1)$-dovan sequence characteristic polynomial can be similarly calculated as performed for Tridovan and Tetradovan.

Definition 2.4. The Z-dovan (Generalization) Characteristic polynomial, where $z$ is the extended sequence order and $Z=z-1$, is:

$$
P_{Z(x)}=x^{(z)}=x^{(z-2)}+x^{(z-3)}+x^{(z-4)}+\ldots+x^{(z-z)} .
$$

It is important to note the inexistence of an element $\left(x^{(z-2)}\right)$ in these equations, validating the recurrence formula of these sequences.

There are some methods that make it easier to obtain the roots, and there is a new method studied in the work of Ozdemir [10], where through a matrix and with the help of hybrid numbers, one can calculate the roots of an equation using Moivre's formula.

\section{Matrix representation}

One way to obtain the linear and recurrent sequence terms is through its generator matrix, where raising to certain powers, its elements are generated. Thus, through investigative studies on Padovan [13] generator matrices, another work defined Tridovan and Tetradovan generator matrices [17].

Theorem 3.1. For $n \in \mathbb{N}$, with $Q_{3}(i, j)$ the term of the matrix $Q_{3}$ represented by the $i$-th line and the $j$-th column, the Tridovan generator matrix $Q_{3}$ is given by:

$$
\text { For } Q_{3}=\left[\begin{array}{llll}
0 & 1 & 0 & 0 \\
1 & 0 & 1 & 0 \\
1 & 0 & 0 & 1 \\
1 & 0 & 0 & 0
\end{array}\right] \text {, have up } Q_{3}^{n}=\left[\begin{array}{llll}
Q_{3}(1,1) & Q_{3}(1,2) & Q_{3}(1,3) & Q_{3}(1,4) \\
Q_{3}(2,1) & Q_{3}(2,2) & Q_{3}(2,3) & Q_{3}(2,4) \\
Q_{3}(3,1) & Q_{3}(3,2) & Q_{3}(3,3) & Q_{3}(3,4) \\
Q_{3}(4,1) & Q_{3}(4,2) & Q_{3}(4,3) & Q_{3}(4,4)
\end{array}\right], n \geqslant 6 \text {. }
$$

In order to simplify the previous matrix terms visualization, calculation of the terms is presented in each of the following lines:

$$
\begin{aligned}
& Q_{3}(1,1)=P_{3(n)}, \\
& Q_{3}(1,2)=P_{3(n-1)}, \\
& Q_{3}(1,3)=P_{3(n-2),} \\
& Q_{3}(1,4)=P_{3(n-3),} \\
& Q_{3}(2,1)=P_{3(n-1)}+P_{3(n-2)}+P_{3(n-3),}, \\
& Q_{3}(2,2)=P_{3(n-2)}+P_{3(n-3)}+P_{3(n-4),}, \\
& Q_{3}(2,3)=P_{3(n-3)}+P_{3(n-4)}+P_{3(n-5),}, \\
& Q_{3}(2,4)=P_{3(n-4)}+P_{3(n-5)}+P_{3(n-6),}, \\
& Q_{3}(3,1)=P_{3(n-1)}+P_{3(n-2)}, \\
& Q_{3}(3,2)=P_{3(n-2)}+P_{3(n-3)}, \\
& Q_{3}(3,3)=P_{3(n-3)}+P_{3(n-4)}, \\
& Q_{3}(3,4)=P_{3(n-4)}+P_{3(n-5),} \\
& Q_{3}(4,1)=P_{3(n-1),}
\end{aligned}
$$


$Q_{3}(4,2)=P_{3(n-2)}$,

$Q_{3}(4,3)=P_{3(n-3)}$,

$Q_{3}(4,4)=P_{3(n-4)}$.

Proof. Using the finite induction principle, one can validate the theorem [17].

Theorem 3.2. For $n \in \mathbb{N}$, with $Q_{4}(i, j)$ the term of the matrix $Q_{4}$ represented by the $i$-th line and $j$-th column, the Tetradovan generator matrix $Q_{4}$ is given by [17]:

For $Q_{4}=\left[\begin{array}{lllll}0 & 1 & 0 & 0 & 0 \\ 1 & 0 & 1 & 0 & 0 \\ 1 & 0 & 0 & 1 & 0 \\ 1 & 0 & 0 & 0 & 1 \\ 1 & 0 & 0 & 0 & 0\end{array}\right]$, have up $Q_{4}^{n}=\left[\begin{array}{lllll}Q_{4}(1,1) & Q_{4}(1,2) & Q_{4}(1,3) & Q_{4}(1,4) & Q_{4}(1,5) \\ Q_{4}(2,1) & Q_{4}(2,2) & Q_{4}(2,3) & Q_{4}(2,4) & Q_{4}(2,5) \\ Q_{4}(3,1) & Q_{4}(3,2) & Q_{4}(3,3) & Q_{4}(3,4) & Q_{4}(3,5) \\ Q_{4}(4,1) & Q_{4}(4,2) & Q_{4}(4,3) & Q_{4}(4,4) & Q_{4}(4,5) \\ Q_{4}(5,1) & Q_{4}(5,2) & Q_{4}(5,3) & Q_{4}(5,4) & Q_{4}(5,5)\end{array}\right]$,

$n \geqslant 8$ and where each term of the previous matrix is given by:

$$
\begin{aligned}
& Q_{4}(1,1)=P_{4(n)}, \\
& Q_{4}(1,2)=P_{4(n-1)}, \\
& Q_{4}(1,3)=P_{4(n-2)}, \\
& Q_{4}(1,4)=P_{4(n-3)}, \\
& Q_{4}(1,5)=P_{4(n-4)} \\
& Q_{4}(2,1)=P_{4(n-1)}+P_{4(n-2)}+P_{4(n-3)}+P_{4(n-4)}, \\
& Q_{4}(2,2)=P_{4(n-2)}+P_{4(n-3)}+P_{4(n-4)}+P_{4(n-5)}, \\
& Q_{4}(2,3)=P_{4(n-3)}+P_{4(n-4)}+P_{4(n-5)}+P_{4(n-6)}, \\
& Q_{4}(2,4)=P_{4(n-4)}+P_{4(n-5)}+P_{4(n-6)}+P_{4(n-7)}, \\
& Q_{4}(2,5)=P_{4(n-5)}+P_{4(n-6)}+P_{4(n-7)}+P_{4(n-8)}, \\
& Q_{4}(3,1)=P_{4(n-1)}+P_{4(n-2)}+P_{4(n-3)}, \\
& Q_{4}(3,2)=P_{4(n-2)}+P_{4(n-3)}+P_{4(n-4)}, \\
& Q_{4}(3,3)=P_{4(n-3)}+P_{4(n-4)}+P_{4(n-5)}, \\
& Q_{4}(3,4)=P_{4(n-4)}+P_{4(n-5)}+P_{4(n-6),}, \\
& Q_{4}(3,5)=P_{4(n-5)}+P_{4(n-6)}+P_{4(n-7)}, \\
& Q_{4}(4,1)=P_{4(n-1)}+P_{4(n-2),} \\
& Q_{4}(4,2)=P_{4(n-2)}+P_{4(n-3)}, \\
& Q_{4}(4,3)=P_{4(n-3)}+P_{4(n-4)}, \\
& Q_{4}(4,4)=P_{4(n-4)}+P_{4(n-5)}, \\
& Q_{4}(4,5)=P_{4(n-5)}+P_{4(n-6),}, \\
& Q_{4}(5,1)=P_{4(n-1)}, \\
& Q_{4}(5,2)=P_{4(n-2)}, \\
& Q_{4}(5,3)=P_{4(n-3)}, \\
& Q_{4}(5,4)=P_{4(n-4)}, \\
& Q_{4}(5,5)=P_{4(n-5)} .
\end{aligned}
$$

Proof. Using the finite induction principle, the theorem validity is demonstrated, [17].

Thus, it can be seen that for an $z$ order extension, following the aforementioned theorems, one can achieve the following corollary. 
Corollary 3.3. The $Q_{Z} Z$-dovan generator matrix, with $n \geqslant 2 z-2, n \in \mathbb{N}$ and $Z=z-1$, is obtained by:

For $Q_{Z}=\left[\begin{array}{cc}0 & {[\boldsymbol{I}]} \\ \mathbf{1} & \mathbf{0}\end{array}\right]$, have up $Q_{Z}^{n}=\left[\begin{array}{cccccc}P_{Z(n)} & \cdots & P_{Z(n-j+1)} & \cdots & P_{Z(n-z+1)} \\ \vdots & \ddots & \vdots & \ddots & \vdots \\ \sum_{i=1}^{z-i+1} P_{Z(n-i)} & \cdots & \sum_{i=1}^{z-i+1} P_{Z(n-i-j+1)} & \cdots & \sum_{i=1}^{z-i+1} P_{Z(n-i-z+1)} \\ \vdots & \ddots & \vdots & \ddots & \vdots \\ P_{Z(n-1)} & \cdots & P_{Z(n-j)} & \cdots & P_{Z(n-z)}\end{array}\right]$.

Where 1 is the column vector and 0 the row vector, both having size $z-1$, the block matrix $[I]$ given by the $z-1$ order identity matrix, $z$ the sequence order, and $i$ and $j$ the row and column indexes where $i \in\{1, \ldots, z\}$ and $j \in\{1, \ldots, z\}$.

Proof. Using the finite induction principle, we have:

For a sequence of order 3 , since the Padovan sequence starts in order 3 , we have $z=3$ :

$$
Q_{2}=\left[\begin{array}{cc}
0 & {[\mathbf{I}]} \\
\mathbf{1} & \mathbf{0}
\end{array}\right]=\left[\begin{array}{lll}
0 & 1 & 0 \\
1 & 0 & 1 \\
1 & 0 & 0
\end{array}\right]
$$

Thus, for $n=4$ we have:

$$
Q_{2}=\left[\begin{array}{lll}
0 & 1 & 0 \\
1 & 0 & 1 \\
1 & 0 & 0
\end{array}\right]^{4}=\left[\begin{array}{lll}
1 & 1 & 1 \\
2 & 1 & 1 \\
1 & 1 & 0
\end{array}\right]=\left[\begin{array}{ccc}
P_{2(4)} & P_{2(3)} & P_{2(2)} \\
P_{2(3)}+P_{2(2)} & P_{2(1)}+P_{2(2)} & P_{2(1)}+P_{2(0)} \\
P_{2(3)} & P_{2(2)} & P_{2(1)}
\end{array}\right]
$$

Therefore, the equality is valid, as shown in the work of Vieira and Alves [17] for the Padovan sequence, where $P_{2(n)}$ represents a Padovan term.

Assuming it is valid for $z=k, k \in \mathbb{N}$, we have:

For $Q_{k-1}=\left[\begin{array}{cc}0 & {[\mathbf{I}]} \\ 1 & 0\end{array}\right]$ that: $Q_{k-1}^{n}\left[\begin{array}{ccccc}P_{k-1(n)} & \ldots & P_{k-1(n-j+1)} & \ldots & P_{k-1(n-k+1)} \\ \vdots & \ddots & \vdots & \ddots & \vdots \\ \sum_{i=1}^{k-i+1} P_{k-1(n-i)} & \ldots \sum_{i=1}^{k-i+1} & P_{k-1(n-i-j+1)} & \ldots \sum_{i=1}^{k-i+1} P_{k-1(n-i-k+1)} \\ \vdots & \ddots & \vdots & \ddots & \vdots \\ P_{k-1(n-1)} & \ldots & P_{k-1(n-j)} & \ldots & P_{k-1(n-k)}\end{array}\right]$.

Now checking for $z=k+1$, we have:

$$
\text { For } Q_{k}=\left[\begin{array}{cc}
0 & {[\mathbf{I}]} \\
\mathbf{1} & \mathbf{0}
\end{array}\right] \text { that: } Q_{k}^{n}\left[\begin{array}{ccccc}
P_{k(n)} & \cdots & P_{k(n-j+1)} & \cdots & P_{k(n-k+2)} \\
\vdots & \ddots & \vdots & \ddots & \vdots \\
\sum_{i=1}^{k-i+2} P_{k(n-i)} & \cdots & \sum_{i=1}^{k-i+2} P_{k(n-i-j+1)} & \cdots & \sum_{i=1}^{k-i+2} P_{k(n-i-k+2)} \\
\vdots & \ddots & \vdots & \ddots & \vdots \\
P_{k(n-1)} & \cdots & P_{k(n-j)} & \cdots & P_{k(n-k+1)}
\end{array}\right] \text {. }
$$


It is noteworthy that the size of the matrix is related to the order of the sequence, thus, it is observed that for Padovan generator matrix there is a $3 \times 3$ matrix, for Tridovan a $4 \times 4$ matrix, up to $Z$-dovan as being $z \times z$ and $Z=z-1$.

\section{Generating function}

The generating function makes it possible to know the terms of a recurring and linear sequence with constant coefficients [9]. This procedure demonstrates how one can find the terms of a recurrent and linear sequence without calculating the previous terms. Thus, there is a formal series where their coefficients carry information about a succession of $a_{n}$ as the function in Equation (3) shows:

$$
g\left(a_{n}, x\right)=\sum_{n=0}^{\infty} a_{n} x^{n}=a_{0} x^{0}+a_{1} x^{1}+a_{2} x^{2}+\ldots
$$

Ferreira [5] studied the generating function of the Padovan sequence. However, Vieira and Alves [17] changed the initial values of this sequence to those of the original definition. Thus, in order to generalize the extension of these numbers, the generating function studied in the work of Vieira and Alves [17] will be used.

Theorem 4.1. The Padovan sequence generating function is given by [5]:

$$
g\left(P_{n}, x\right)=\frac{1}{1-x^{2}-x^{3}}
$$

Proof. This is a formal series in which its coefficients get information about a $a_{n}$ sequence with $n$ belonging to the natural numbers, and being a $g\left(a_{n}, x\right)$ function defined by the series:

$$
g\left(a_{n}, x\right)=\sum_{n=0}^{\infty} a_{n} x^{n}=a_{0} x^{0}+a_{1} x^{1}+a_{2} x^{2}+\ldots
$$

This function is multiplied by $x^{2}, x^{3}$ in the equations below because of its recurrence relationship.

So for Padovan, with his shifted starting values equal to $P_{0}=1, P_{1}=0, P_{2}=1$, we have:

$$
\begin{aligned}
g\left(P_{(n)}, x\right) & =P_{0}+P_{1} x+P_{2} x^{2}+P_{3} x^{3}+P_{4} x^{4}+\ldots \\
x^{2} g\left(P_{(n)}, x\right) & =P_{0} x^{2}+P_{1} x^{3}+P_{2} x^{4}+P_{3} x^{5}+P_{4} x^{6}+\ldots \\
x^{3} g\left(P_{(n)}, x\right) & =P_{0} x^{3}+P_{1} x^{4}+P_{2} x^{5}+P_{3} x^{6}+P_{4} x^{7}+\ldots
\end{aligned}
$$

based on Equation 4 - (5+6), we have:

$$
\begin{aligned}
g\left(P_{n}, x\right)\left(1-x^{2}-x^{3}\right) & =P_{0}+P_{1} \cdot x+\left(P_{2}-P_{0}\right) x^{2} \\
g\left(P_{n}, x\right)\left(1-x^{2}-x^{3}\right) & =1 \\
g\left(P_{n}, x\right) & =\frac{1}{1-x^{2}-x^{3}} .
\end{aligned}
$$

Theorem 4.2. Tridovan generating function, $P_{3(n)}$, for positive terms is [17]:

$$
g\left(P_{3(n)}, x\right)=\sum_{n=0}^{\infty} P_{3(n)} x=\frac{1}{\left(1-x^{2}-x^{3}-x^{4}\right)}
$$

Proof. Similar to the proof of Theorem 4.1. 
Theorem 4.3. Tetradovan generating function, $P_{4(n)}$, for positive terms is [17]:

$$
g\left(P_{4(n)}, x\right)=\sum_{n=0}^{\infty} P_{4(n)} x=\frac{1}{\left(1-x^{2}-x^{3}-x^{4}-x^{5}\right)}
$$

Proof. Similar to the proof of Theorem 4.1.

Thus, from Theorem 4.1, Theorem 4.2 and Theorem 4.3, one can define the generalization of the Generating Function to a $z$ ( $Z$-dovan), where $Z=z-1$.

Corollary 4.4. The z order sequence generating function Z-dovan is defined as:

$$
g\left(P_{Z(n)}, x\right)=\frac{1}{1-\sum_{i=2}^{z} x^{i}}
$$

where $z$ represents the sequence order, ranging from 3 to $z$ and $Z=z-1$.

Proof. Using the finite induction principle, we have:

For $z=3$, we have:

$$
g\left(P_{2(n)}, x\right)=\frac{1}{1-\sum_{i=2}^{3} x^{i}}=\frac{1}{1-\left(x^{2}+x^{3}\right)}=\frac{1}{1-x^{2}-x^{3}}
$$

As seen and validated in Theorem 4.1 for the Padovan sequence of order 3.

Assuming it is valid for any $z=k, k \in \mathbb{N}$, we have:

$$
g\left(P_{k-1(n)}, x\right)=\frac{1}{1-\sum_{i=2}^{k} x^{i}}=\frac{1}{1-\left(x^{2}+x^{3}+\ldots+x^{k}\right)}
$$

With this, verifying that it is valid for $z=k+1$, we have:

$$
g\left(P_{k(n)}, x\right)=\frac{1}{1-\sum_{i=2}^{k+1} x^{i}}=\frac{1}{1-\left(x^{2}+x^{3}+\ldots+x^{k+1}\right)}
$$

with initial values already set.

\section{Conclusion}

Initially, from studies concerning the Tridovan and Tetradovan sequences, it was possible to carry out an investigation and thus obtain the Padovan sequence generator matrix generalization of order $z$. The generating function and characteristic polynomial generalization of these numbers under analysis was established. It is noteworthy that this process was presented in a primordial way in this work, thus introducing a new generalized sequence numbers calculating way of $z$ order, without needing to know previous terms.

For future work, we investigate the application of these matrices in the computational area, as well as their use in economic and architectural processes [2-4]. 


\section{References}

[1] Alsina, C., \& Nelsen, R. B. (2015). A Mathematical Space Odyssey: Solid Geometry in the 21th Century. American Mathematical Society; UK ed., Washington.

[2] Deveci, O. (2018). The Padovan-Circulant Sequences and their Applications. Mathematical Reports, 20 (70), 401-416.

[3] Deveci, O., \& Karaduman, E. (2017). On The Padovan p-Numbers. Hacettepe Journal of Mathematics and Statistics, 46 (4), 579-592.

[4] Deveci, O., \& Shannon, A. G. (2017). Pell-Padovan-circulant sequences and their applications. Notes on Number Theory and Discrete Mathematics, 23(3), 100-114.

[5] Ferreira, R. de C. (2015). Números Mórficos. 2015. 94 f. Dissertação (Mestrado Profissional em Matemática em Rede Nacional PROFMAT) - Universidade Federal da Paraíba, Paraíba.

[6] Gomes, C. A., \& Oliveira, O. R. B. (2019). O Teorema de Cayley-Hamilton. Available online:https://www.ime.usp.br/ oliveira/ELE-Cayley-Hamilton.pdf

[7] Gomes, V. E. D., \& Martelo, M. A. P. (2017). Formato da solução geral das sequências recorrentes lineares homogêneas com coeficientes constantes. Revista Eletrônica da Matemática - REMAT, 3, 159-173.

[8] Kilic, E., \& Tasci, D. (2006). On the Generalized Order- $k$ Fibonacci and Lucas Numbers. Rocky Mountain Journal of Mathematics, 36, 1915-1926.

[9] Koshy, T. (2001) Fibonacci and Lucas Numbers with Applications. Wiley Interscience, New York.

[10] Ozdemir, M. (2019). Finding $n$-th Roots of a $2 \times 2$ Real Matrix Using De Moivre's Formula. Advances in Applied Clifford Algebras, 29, Article No. 2.

[11] Padovan, R. (2002). Dom hans van der laan and the plastic number. Nexus Network Journal, 4, 181-193.

[12] Silva, A. K. S. (2018). Determinação de uma fórmula para calcular os termos de uma sequência de Fibonacci por meio de progressões geométricas e espaços vetoriais. Revista Eletrônica da Matemática - REMAT, 4, 87-97.

[13] Sokhuma, K. (2013). Padovan $q$-matrix and the generalized relations. Applied Mathematical Sciences, 7(01), 2777-2780.

[14] Stakhov, A. P. (1999). A generalization of the Fibonacci Q-matrix. Reports of the National Academy of Sciences of Ukraine, 9, 46-49.

[15] Stewart, I. (1996). Tales of a Neglected Number. Scientific American, 274(6), 102-103. 
[16] Tasci, D., \& Kilic, E. (2004). On the order- $k$ generalized Lucas numbers. Applied Mathematics and Computation, 153, 637-641.

[17] Vieira, R. P. M., \& Alves, F. R. V. (2019). Propriedades das extensões da Sequência de Padovan. C.Q.D.-Revista Eletrônica Paulista de Matemática, 15, 24-40, Edição Iniciação Científica.

[18] Vieira, R. P. M., \& Alves, F. R. V. (2019). Sequences of Tridovan and their identities. Notes on Number Theory and Discrete Mathematics, 25(3), 185-197.

[19] Voet, C., \& Schoonjans, Y. (2012). Benedictine thought as a catalist for 20st century liturgical space: the motivation behind Dom Hans van der Laan's aesthetic church arquitectury. In: Proceeding of the 2nd international Conference of the European Architetural History of Network, 255-261.

[20] Yilmaz, N., \& Taskara, N. (2013). Binomial Transforms of the Padovan and Perrin Matrix Sequences. Abstract and Applied Analysis, 2013, Article ID 497418, 1-7. 